\title{
A New Approach for Hypertension: the Case of Sacubitril/ Valsartan Experienced in Randomized Controlled Trials That Selectively Restrict Its Use to the Hypertension of the Elderly
}

\author{
Renato De Vecchis ${ }^{\mathrm{a}, \mathrm{c}}$, Carmelina Ariano ${ }^{\mathrm{b}}$, Silvia Soreca ${ }^{\mathrm{a}}$
}

Sacubitril/valsartan, a conjugation molecule containing a neprilysin inhibitor (sacubitril) and an angiotensin receptor blocker (valsartan), has been approved in Europe since March 2017 for the treatment of chronic heart failure with reduced left ventricular ejection fraction (HFREF) in NYHA class II-III.

There are some relatively little-known data about neprilysin inhibition in the mammalian brain. In particular, it is known that also cerebral beta-amyloid, that has polypeptide structure like atrial natriuretic factor and B-type natriuretic peptide, is mainly degraded by neprilysin [1]. Thus, sacubitril/ valsartan has been accused of propitiating an increase in betaamyloid concentration in cerebrospinal fluid [2]. In this regard, it is well known that abnormally high amounts of beta-amyloid have been found in the brain of patients with Alzheimer's disease.

Overall, the studies about the relationship between sacubitril/valsartan and possible neurotoxic side-effects [3, 4] would seem to exclude that usual therapeutic doses of sacubitril/valsartan might produce neuro- or psycho-toxic effects.

However, the impression remains that the manufacturing company has deliberately avoided proposing the extension of therapeutic indications of sacubitril/valsartan to hypertension, considering it appropriate to temporarily limit the official indication of the drug only to HFREF patients. In fact they are often characterized by a relatively high mean age ( $>70$ years) with the inherent implication that any deficit of cognitive functions occurring at this age could be reasonably ascribed to senile involution of mental faculties. Instead the codification of sacubitril/valsartan as a drug indicated for hypertension would involve the responsibility of intervening also in the case of young patients with the prospect to carry out a continuous, over several decades treatment burdened by the risk of a pos-

Manuscript submitted August 25, 2018, accepted September 11, 2018

aPreventive Cardiology and Rehabilitation Unit, DSB 29 "S. Gennaro dei Poveri Hospital”, via S.Gennaro dei Poveri 25, 80136 Napoli, Italy

bDivision of Geriatrics, "Casa Sollievo della Sofferenza" Hospital, viale Cappuccini 2, 71013 San Giovanni Rotondo, Italy

${ }^{\mathrm{c} C}$ Corresponding Author: Renato De Vecchis, Preventive Cardiology and Rehabilitation Unit, DSB 29 "S. Gennaro dei Poveri Hospital", via S.Gennaro dei Poveri 25, 80136 Napoli, Italy. Email: devecchis.erre@virgilio.it sible harmful effect on the cognitive functions favored by the long duration of exposure to the toxic agent [5].

This has also entailed that the investigational use of sacubitril/valsartan as an antihypertensive agent has been deliberately circumscribed to elderly. We found a total of five randomized controlled trials (RCTs) centered on the antihypertensive effect of sacubitril/valsartan. In these RCTs, overall enrolling 1,513 patients, sacubitril/valsartan was compared with olmesartan (three studies) [6-8] or valsartan (two studies) $[9,10]$ and tested exclusively in the elderly (mean age about 60 years). In other words, these RCTs were conceived to investigate the use of sacubitril/valsartan for isolated elderly systolic hypertension and all demonstrated significant superiority of the antihypertensive effect of sacubitril/valsartan compared to olmesartan or valsartan. However, selection made with the exclusion of young hypertensives is likely to have been caused by concerns of defensive medicine. In fact, in the PARADIGM-HF study the hypotensive effect of the molecule had occurred with the same intensity in both relatively young and elderly patients.

Thus, perplexities and prejudices related to the supposed neurotoxic effects of sacubitril/valsartan have made it reserved for: 1) patients with HFREF, NHYA class II-III (indication 1A), for whom life expectancy is reduced and the high risk of impending hemodynamic deterioration requires to use the best evidence-based therapy available; and 2) exclusively within RCTs, for old, i.e., aged 60 years or over, hypertensives in whom any occurrence of "mild cognitive impairment" could easily be attributed to the hypertension itself. Therefore a large group of patients, i.e., hypertensives aged between 20 and 60 years, remains excluded from the combination therapy with neprilysin inhibitor plus angiotensin receptor blocker. It is hoped that the progress of knowledge in neuro-physiopathology will lead to the definitive denial of the supposed neurotoxic effects of neprilysin inhibition, so as to make possible the access to the benefits of sacubitril/valsartan even for those currently excluded for reasons of caution and "defensive medicine".

\section{Conflict of Interest}

Renato De Vecchis, Carmelina Ariano and Silvia Soreca do not 
have any conflict of interest to declare.

\section{Funding Support}

There are no sources of funding and/or financial support for this manuscript.

\section{References}

1. Guan H, Liu Y, Daily A, Police S, Kim MH, Oddo S, LaFerla FM, et al. Peripherally expressed neprilysin reduces brain amyloid burden: a novel approach for treating Alzheimer's disease. J Neurosci Res. 2009;87(6):1462-1473.

2. Campbell DJ. Long-term neprilysin inhibition - implications for ARNIs. Nat Rev Cardiol. 2017;14(3):171-186.

3. Langenickel TH, Tsubouchi C, Ayalasomayajula S, Pal $\mathrm{P}$, Valentin MA, Hinder M, Jhee S, et al. The effect of LCZ696 (sacubitril/valsartan) on amyloid-beta concentrations in cerebrospinal fluid in healthy subjects. Br J Clin Pharmacol. 2016;81(5):878-890.

4. Schoenfeld HA, West T, Verghese PB, Holubasch M, Shenoy N, Kagan D, Buono C, et al. The effect of angiotensin receptor neprilysin inhibitor, sacubitril/valsartan, on central nervous system amyloid-beta concentrations and clearance in the cynomolgus monkey. Toxicol Appl Pharmacol. 2017;323:53-65.

5. Ruiz-Hurtado G, Ruilope LM. Advantages of sacubitril/ valsartan beyond blood pressure control in arterial hypertension. Eur Heart J. 2017;38(44):3318-3320.

6. Williams B, Cockcroft JR, Kario K, Zappe DH, Brunel PC, Wang Q, Guo W. Effects of sacubitril/valsartan versus olmesartan on central hemodynamics in the elderly with systolic hypertension: the PARAMETER study. Hypertension. 2017;69(3):411-420.

7. Supasyndh O, Wang J, Hafeez K, Zhang Y, Zhang J, Rakugi H. Efficacy and safety of Sacubitril/Valsartan (LCZ696) compared with Olmesartan in elderly Asian patients $(>/=65$ Years $)$ with systolic hypertension. Am J Hypertens. 2017;30(12):1163-1169.

8. Schmieder RE, Wagner F, Mayr M, Delles C, Ott C, Keicher C, Hrabak-Paar M, et al. The effect of sacubitril/valsartan compared to olmesartan on cardiovascular remodelling in subjects with essential hypertension: the results of a randomized, double-blind, active-controlled study. Eur Heart J. 2017;38(44):3308-3317.

9. Izzo JL, Jr., Zappe DH, Jia Y, Hafeez K, Zhang J. Efficacy and safety of crystalline Valsartan/Sacubitril (LCZ696) compared with placebo and combinations of free valsartan and sacubitril in patients with systolic hypertension: the RATIO study. J Cardiovasc Pharmacol. 2017;69(6):374-381.

10. Wang TD, Tan RS, Lee HY, Ihm SH, Rhee MY, Tomlinson B, Pal P, et al. Effects of Sacubitril/Valsartan (LCZ696) on natriuresis, diuresis, blood pressures, and NT-proBNP in salt-sensitive hypertension. Hypertension. 2017;69(1):32-41. 